\title{
$\pi B_{8} B_{8}$ and $\sigma B_{8} B_{8}$ couplings from a chiral quark potential model
}

\author{
M.T. Fernández-Caramés and P. González \\ Departamento de Física Teórica e IFIC, \\ Universidad de Valencia - CSIC, E-46100 Burjassot, Valencia, Spain
}

\author{
A. Valcarce \\ Departamento de Física Fundamental, \\ Universidad de Salamanca, E-37008 Salamanca, Spain
}

(Dated: May 9, 2022)

\begin{abstract}
From an $\mathrm{SU}(2) \otimes \mathrm{SU}(2)$ chiral quark potential model incorporating spontaneous chiral symmetry breaking the asymptotic $\pi$ and $\sigma$ exchange pieces of the $N N$ potential are generated. From them the $\pi N N$ and $\sigma N N$ coupling constants can be extracted. The generalization to $\mathrm{SU}(3) \otimes \mathrm{SU}(3)$ allows for a determination of $\pi B_{8} B_{8}$ and $\sigma B_{8} B_{8}$ coupling constants according to exact $\mathrm{SU}(3)$ hadron symmetry. The implementation of the values of the couplings at $Q^{2}=0$ provided by QCD sum rules and/or phenomenology makes also feasible the extraction of the meson-baryon-baryon form factors. In this manner a quite complete knowledge of the couplings may be attained.
\end{abstract}

PACS numbers: 12.39.Jh, 14.20.-c, 14.20.Gk 


\section{INTRODUCTION}

The meson-baryon-baryon $\left(m B B^{\prime}\right)$ couplings play a central role in hadron physics concerning the baryon-baryon $\left(B B^{\prime}\right)$ interactions as well as the formation and decay of baryon resonances. To study these couplings effective hadron lagrangians involving the mesons and baryons under consideration are postulated. All the complexity of the $m B B^{\prime}$ vertices is assumed to be taken into account through running couplings depending on $Q^{2}$, the transfer momentum in the vertex. This dependency is usually parametrized in terms of a form factor and a coupling constant defined as the value of the running coupling at a particular $Q^{2}$, usually on-shell $Q^{2}=M_{m}^{2}$. Then one can calculate physical processes and compare to data to extract these values. Thus the $\pi N N$ coupling constant is obtained from $\pi N$ and/or $N N$ scattering data [1]. For couplings involving baryons and/or mesons for which scattering or decay data are not so complete or unavailable one can also rely on symmetry to derive predictions, see for instance [2].

From a more fundamental point of view hadrons are made up of quarks. Hence hadron structures and decays as well as hadron-hadron interactions should come out from quark dynamics as dictated by QCD. Due to the technical difficulty to achieve this objective at present quark models of hadrons incorporating QCD-motivated symmetries and dynamics have been successfully applied to generate the baryon-baryon interactions, and consistently the baryon spectrum, in the light $(u, d)$ [3] as well as in the light + strange $(u, d, s)$ quark sectors [4, 5, 6]. These models, sometimes less precise than effective hadronic treatments, offer the advantage of providing a consistent unified description of all baryon-baryon processes from the same hamiltonian at the quark level. This confers them in principle a great predictive power once the model parameters are tightly constrained from some selected set of existing data.

We shall make use of this power to predict, within a non-relativistic chiral quark model framework, $m B_{8} B_{8}$ ( $B_{8}$ : baryon of the flavor octet) coupling constants in terms of mesonquark-quark $(m q q)$ couplings. More precisely we shall generate, from a $m q q$ lagrangian incorporating the effect of spontaneous chiral symmetry breaking (SCSB), the quark-quark meson exchange potentials and from them, through a Born-Oppenheimer (BO) approximation, the asymptotic baryon-baryon meson exchange interactions. The use of justified harmonic oscillator baryon wave functions (in terms of quarks) will allow us to perform analytic calculations. By comparing the resulting interactions to the ones postulated at the effective hadronic level we shall identify the meson-baryon-baryon coupling constants. This procedure has been applied in the literature to the $\pi N N$ coupling [7]. Here we shall be more precise in the extraction of coupling constants and form factors and we shall extend its application to the $\sigma$ meson and the other $B_{8}$ baryons making feasible the comparison of our results to the ones obtained with alternative methods based on quark or hadron degrees of freedom.

The contents are organized as follows. In Sect. II we shall center on the light quark sector where spontaneously broken chiral $\mathrm{SU}(2) \otimes \mathrm{SU}(2)$ symmetry serves as an underlying general guide to generate the quark-quark meson-exchange potentials. We shall revisit the calculation of the $\pi N N$ coupling in terms of the $\pi q q$ one and apply the same procedure to the $\sigma N N$ case. We shall also comment on the possibility of applying our method to $\Delta$ and nucleon resonances. Then in Sect. III we shall consider the extension, via chiral $\mathrm{SU}(3) \otimes \mathrm{SU}(3)$, to the $\mathrm{SU}(3)$ octet of baryons. Finally in Sect. IV] we shall summarize our main results and conclusions. 


\section{LIGHT QUARK SECTOR: $\mathrm{SU}(2) \otimes \mathrm{SU}(2)$.}

Our starting point is the chiral lagrangian

$$
\mathcal{L}_{c h}=-g_{c h} \bar{q}\left(\mathbb{1}_{S U(2)} \sigma+i \gamma_{5} \vec{\tau} \vec{\pi}\right) q,
$$

where $q$ has components $u$ and $d$, and $g_{c h}$ is the chiral mqq coupling constant $(m: \pi, \sigma)$. The spontaneous chiral symmetry breaking gives rise to a vertex form factor $F\left(Q^{2}\right)$ [8]. From the parametrization given in references [9, 10] we propose the Lorentz invariant form (note that for the purpose of the derivation of static potentials $Q^{2}=-\vec{Q}^{2}$ ),

$$
F\left(Q^{2}\right)=\left(\frac{\Lambda^{2}}{\Lambda^{2}-Q^{2}}\right)^{1 / 2},
$$

where $\Lambda$ is an effective cutoff parameter fitted from data. We should keep in mind that this parametrization of $F\left(Q^{2}\right)$ makes only sense for $Q^{2}<\Lambda^{2}$.

From the form proposed $\left(F\left(Q^{2}=0\right)=1\right)$ it is clear that $g_{c h}$ represents the value of the $m q q$ coupling, $g_{c h} F\left(Q^{2}\right)$, at $Q^{2}=M_{m}^{2}$ in the limit $M_{m}^{2}=0$. In order to deal with coupling constants defined on the physical meson masses $\left(M_{\pi}^{2} \neq 0 \neq M_{\sigma}^{2}\right)$ we identify (it is implicitly assumed that $M_{\pi}^{2}, M_{\sigma}^{2}<\Lambda^{2}$ )

$$
\begin{aligned}
g_{\pi q q} & \equiv g_{c h}\left(\frac{\Lambda^{2}}{\Lambda^{2}-M_{\pi}^{2}}\right)^{1 / 2} \\
g_{\sigma q q} & \equiv g_{c h}\left(\frac{\Lambda^{2}}{\Lambda^{2}-M_{\sigma}^{2}}\right)^{1 / 2},
\end{aligned}
$$

as the values of the coupling $g_{c h} F\left(Q^{2}\right)$ at $Q^{2}=M_{\pi}^{2}$ and $Q^{2}=M_{\sigma}^{2}$, respectively.

Let us point out that the use of the experimental pion mass may have required the introduction of an explicit symmetry breaking term proportional to $\sigma$ in the lagrangian but this term has not any further effect in the analysis we perform. Regarding the $\sigma$ mass it will be taken as a parameter to be fitted from data around the value provided by the SCSB relation [11],

$$
M_{\sigma}^{2}-M_{\pi}^{2}=4 M_{q}^{2}
$$

where $M_{q}$ denotes the constituent quark mass at $Q^{2}=0$.

In terms of $g_{\pi q q}$ we write the pion lagrangian as

$$
\mathcal{L}_{\pi q q}=-g_{\pi q q} \bar{q} i \gamma_{5} \vec{\tau} \vec{\pi} q
$$

with a vertex form factor $F_{\pi q q}\left(Q^{2}\right)$ given by

$$
F_{\pi q q}\left(Q^{2}\right) \equiv\left(\frac{\Lambda^{2}-M_{\pi}^{2}}{\Lambda^{2}-Q^{2}}\right)^{1 / 2}
$$

Analogously, the sigma lagrangian reads

$$
\mathcal{L}_{\sigma q q}=-g_{\sigma q q} \bar{q} \mathbb{1}_{S U(2)} \sigma q
$$

with a vertex form factor $F_{\sigma q q}\left(Q^{2}\right)$ given by

$$
F_{\sigma q q}\left(Q^{2}\right) \equiv\left(\frac{\Lambda^{2}-M_{\sigma}^{2}}{\Lambda^{2}-Q^{2}}\right)^{1 / 2}
$$

Note that both form factors are of the same type $F_{m q q}\left(Q^{2}\right) \equiv\left(\Lambda^{2}-M_{m}^{2} / \Lambda^{2}-Q^{2}\right)^{1 / 2}$ so that $F_{m q q}\left(Q^{2}=M_{m}^{2}\right)=1$. 


\section{A. $\quad m q q$ and induced $m B B$ potentials}

From $\mathcal{L}_{m q q}$ and the form factors the static OPE and OSE central potentials (to the respective lowest order in $Q^{2}$ ) can be obtained through a non-relativistic reduction of the corresponding Feynman diagram amplitudes. They are

$$
\begin{gathered}
V_{\mathrm{OPE}}^{i j}\left(\vec{r}_{i j}\right)=\frac{1}{3} \frac{g_{\pi q q}^{2}}{4 \pi} \frac{M_{\pi}^{2}}{4 M_{i} M_{j}} M_{\pi}\left[Y\left(M_{\pi} r_{i j}\right)-\frac{\Lambda^{3}}{M_{\pi}^{3}} Y\left(\Lambda r_{i j}\right)\right]\left(\vec{\sigma}_{i} \cdot \vec{\sigma}_{j}\right)\left(\vec{\tau}_{i} \cdot \vec{\tau}_{j}\right), \\
V_{\mathrm{OSE}}^{i j}\left(\vec{r}_{i j}\right)=-\frac{g_{\sigma q q}^{2}}{4 \pi} M_{\sigma}\left[Y\left(M_{\sigma} r_{i j}\right)-\frac{\Lambda}{M_{\sigma}} Y\left(\Lambda r_{i j}\right)\right] .
\end{gathered}
$$

Here $i$ and $j$ are numbers denoting quarks, $M_{i, j}=M_{q}, \vec{\sigma}_{i, j}\left(\vec{\tau}_{i, j}\right)$ are the spin (isospin) Pauli operators, $r_{i j}$ is the interquark distance and the function $Y$ is defined as,

$$
Y(x)=\frac{e^{-x}}{x} .
$$

Once the potentials at the quark level have been derived we shall use them to obtain the baryon-baryon potentials. From $V_{\mathrm{OmE}}^{i j}$ the asymptotic baryon-baryon meson exchange static potential is defined as

$$
\left(V_{q}\right)_{\mathrm{OmE}}^{B_{a} B_{b} \rightarrow B_{c} B_{d}}(R \rightarrow \infty) \equiv \lim _{R \rightarrow \infty}<\Psi_{B_{c} B_{d}}\left|\sum_{\substack{i \in B_{a}, B_{c} \\ j \in B_{b}, B_{d}}} V_{O m E}^{i j}\right| \Psi_{B_{a} B_{b}}>
$$

where $\Psi_{B_{i} B_{j}}$ stands for the two-baryon wave function, $R$ for the interbaryon distance and the integration is over the quark coordinates.

We shall concentrate on $B_{a} B_{b} \rightarrow B_{c} B_{d}$ interactions involving baryons with the same mass. Then the asymptotic two-baryon wave function will be expressed in the center of mass system as

$$
\Psi_{B_{a} B_{b}}=\Phi_{B_{a}}(1,2,3 ;+\vec{R} / 2) \Phi_{B_{b}}(4,5,6 ;-\vec{R} / 2)
$$

where $(1,2,3)$ and $(4,5,6)$ denote the quarks forming the baryons, $\pm \vec{R} / 2$ the baryons position and

$$
\Phi_{B_{i}}=\left(\Phi_{B_{i}}\right)_{\text {spatial }}\left(\Phi_{B_{i}}\right)_{\text {spin-flavor }}\left(\Phi_{B_{i}}\right)_{\text {color }}
$$

is the one-baryon wave function expressed as the direct product of its spatial, spin-flavor

and color parts. For the sake of simplicity the baryon spatial wave function will be chosen of harmonic oscillator type

$$
\left(\Phi_{B}\right)_{\text {spatial }}(1,2,3 ;+\vec{R} / 2)=\prod_{i=1}^{3}\left(\frac{1}{\pi b^{2}}\right)^{\frac{3}{4}} \exp \left[-\left(\vec{r}_{i}-\vec{R} / 2\right)^{2} / 2 b^{2}\right]
$$

with an harmonic oscillator parameter, $b$, related to the size of the baryon.

\section{B. Parameters}

In order to fix the parameters at the quark level: $M_{q}, M_{\pi}, M_{\sigma}, g_{c h}, \Lambda$, and $b$, we shall rely on the efficient description of $N N$ data provided by the Chiral Quark Cluster Model 
(CQCM) [3]. Such model contains, apart from OPE and OSE potentials derived from $\mathcal{L}_{m q q}$, a confinement plus a residual one-gluon exchange (OGE) interactions. We should realize though that the precise fitting of the parameters in the CQCM relies on a RGM calculation so that the two-baryon wave function as well as the baryon-baryon potentials are different from the ones obtained via the Born-Oppenheimer (BO) approach we follow, Eq. (13). We shall take this into account in an effective manner by keeping the same values for $M_{q}, M_{\pi}$, $M_{\sigma}, \Lambda$, and $b$, and fitting a new value for $g_{c h}$ to reproduce, with our BO approach, the experimental value of the pion-nucleon-nucleon coupling constant (see next section). The values of the parameters used henceforth are listed in Table I

TABLE I: Quark model parameters [3].

\begin{tabular}{|c|c|c|c|c|}
\hline $\begin{array}{l}M_{q} \\
(\mathrm{MeV})\end{array}$ & $\begin{array}{l}b \\
(\mathrm{fm})\end{array}$ & $\begin{array}{l}M_{\sigma} \\
\left(\mathrm{fm}^{-1}\right)\end{array}$ & $\begin{array}{l}M_{\pi} \\
\left(\mathrm{fm}^{-1}\right)\end{array}$ & $\begin{array}{l}\Lambda \\
\left(\mathrm{fm}^{-1}\right)\end{array}$ \\
\hline$\overline{313}$ & 0.518 & 3.42 & 0.7 & 4.2 \\
\hline
\end{tabular}

\section{C. $\pi \mathbf{N N}$}

From Eqs. (10), (13) and (16) the asymptotic OPE central potential for $N N \rightarrow N N$, corresponding to the diagram of Fig. 1 (there are nine equivalent ones), can be obtained. It reads (the calculation has been explicitly done in Ref. [7])

$$
\left(V_{q}\right)_{\mathrm{OPE}}^{N N \rightarrow N N}(R \rightarrow \infty)=\frac{g_{\pi q q}^{2}}{4 \pi}\left[9<\left(\vec{\sigma}_{3} \cdot \vec{\sigma}_{6}\right)_{N N} \cdot\left(\vec{\tau}_{3} \cdot \vec{\tau}_{6}\right)_{N N}>e^{\frac{M_{\pi}^{2} b^{2}}{2}}\right] \frac{M_{\pi}^{2}}{4 M_{q}^{2}} \frac{1}{3} \frac{e^{-M_{\pi} R}}{R} .
$$

To extract the $\pi N N$ coupling we have to compare this potential with the one derived from

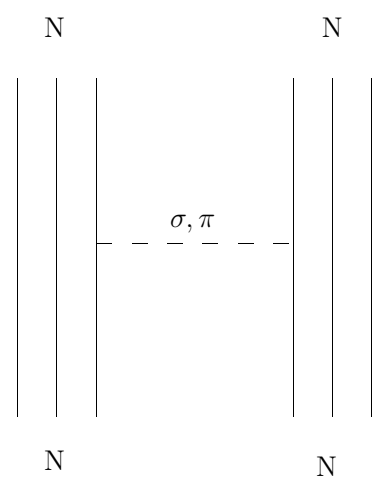

FIG. 1: Asymptotic NN OPE or OSE interactions at quark level.

a postulated hadronic lagrangian. Assuming for instance a pseudoscalar coupling we can write a lagrangian

$$
\mathcal{L}_{\pi N N}=-\left(g_{\pi N N}\right)_{Q^{2}=M_{\pi}^{2}} \bar{N} i \gamma_{5} \vec{\tau} \vec{\pi} N
$$

with a vertex form factor $G_{\pi N N}\left(Q^{2}\right)$ so that $G_{\pi N N}\left(Q^{2}=M_{\pi}^{2}\right)=1$. Notice that we have indicated explicitly the on-shell character of the coupling $g_{\pi N N}$ through the subindex. In order to derive from this lagrangian a Yukawa-like pion exchange potential, monopole or 
dipole type form factors are usually assumed. Concerning the asymptotic potential both give the same result. We shall use in parallel with the form factor at the quark level a form

$$
G_{\pi N N}\left(Q^{2}\right) \equiv\left(\frac{\Lambda_{\pi N N}^{2}-M_{\pi}^{2}}{\Lambda_{\pi N N}^{2}-Q^{2}}\right)^{\frac{1}{2}}
$$

valid for $Q^{2}<\Lambda_{\pi N N}^{2}$ and $M_{\pi}^{2}<\Lambda_{\pi N N}^{2}\left(\Lambda_{\pi N N}\right.$ is a cutoff parameter to be fitted).

Note that if we had preferred to refer the lagrangian to the value of the coupling at $Q^{2}=0$, i.e., to $\left(g_{\pi N N}\right)_{Q^{2}=0}$, then we would have a different form factor such that

$$
\left(g_{\pi N N}\right)_{Q^{2}=M_{\pi}^{2}} G_{\pi N N}\left(Q^{2}\right)=\left(g_{\pi N N}\right)_{Q^{2}=0}\left(\frac{\Lambda_{\pi N N}^{2}}{\Lambda_{\pi N N}^{2}-Q^{2}}\right)^{\frac{1}{2}}
$$

where the second term on the right hand side represents the form factor normalized at $Q^{2}=0$.

From $\mathcal{L}_{\pi N N}$ and $G_{\pi N N}\left(Q^{2}\right)$ the non-relativistic reduction of the one-pion exchange diagram, Fig. 2, to the lowest order in $Q^{2}$, provides us with the pion exchange static potential at the baryonic level. The asymptotic behavior of its central part is given by

$$
\left(\mathcal{V}_{B}\right)_{\mathrm{OPE}}^{N N \rightarrow N N}(R \rightarrow \infty)=\frac{\left(g_{\pi N N}^{2}\right)_{Q^{2}=M_{\pi}^{2}}}{4 \pi}\left[\left\langle\left(\vec{\sigma}_{N} \cdot \vec{\sigma}_{N}\right)\left(\vec{\tau}_{N} \cdot \vec{\tau}_{N}\right)\right\rangle\right] \frac{M_{\pi}^{2}}{4 M_{N}^{2}} \frac{1}{3} \frac{e^{-M_{\pi} R}}{R}
$$

Note that $\left(\mathcal{V}_{B}\right)_{\mathrm{OPE}}^{N N \rightarrow^{N N}}(R \rightarrow \infty)$ only depends on the coupling constant and not on the

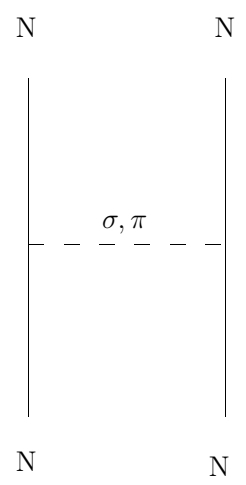

FIG. 2: Asymptotic $N N$ OPE or OSE interactions at baryon level.

form factor. Then no information on the form factors at the baryon level can be extracted from it. Regarding the coupling constant we can make use of the relation $(25 / 9)<\left(\vec{\sigma}_{N}\right.$. $\left.\vec{\sigma}_{N}\right)\left(\vec{\tau}_{N} \cdot \vec{\tau}_{N}\right)>=9<\left(\vec{\sigma}_{3} \cdot \vec{\sigma}_{6}\right)_{N N} \cdot\left(\vec{\tau}_{3} \cdot \vec{\tau}_{6}\right)_{N N}>$ [7] to compare Eqs. (17) and (21). From this comparison we extract

$$
\left(g_{\pi N N}^{2}\right)_{Q^{2}=M_{\pi}^{2}}=g_{\pi q q}^{2} \frac{M_{N}^{2}}{M_{q}^{2}} \frac{25}{9} e^{\frac{M_{\pi}^{2} b^{2}}{2}} .
$$

Having chosen $M_{q}=313 \mathrm{MeV}$ so that $M_{N}=3 M_{q}$ we can re-express

$$
\left(g_{\pi N N}^{2}\right)_{Q^{2}=M_{\pi}^{2}}=25 g_{\pi q q}^{2} e^{\frac{M_{\pi}^{2} b^{2}}{2}} .
$$




\section{Pseudovector couplings}

Alternatively to $g_{\pi q q}$ and $g_{\pi N N}$ we could have used pseudovector couplings $f_{\pi q q}$ and $f_{\pi N N}$ so that

$$
\mathcal{L}_{\pi q q}^{P V}=-\frac{f_{\pi q q}}{M_{\pi}} \bar{q} i \gamma_{5} \gamma_{\mu} \vec{\tau} \partial^{\mu} \vec{\pi} q
$$

with the vertex form factor $F_{\pi q q}\left(Q^{2}\right)$ and

$$
\mathcal{L}_{\pi N N}^{P V}=-\frac{\left(f_{\pi N N}\right)_{Q^{2}=M_{\pi}^{2}}}{M_{\pi}} i \gamma_{5} \gamma_{\mu} \vec{\tau} \partial^{\mu} \vec{\pi} N
$$

with the vertex form factor $G_{\pi N N}\left(Q^{2}\right)$.

It turns out that $\mathcal{L}^{P V}$ give rise to exactly the same potentials as $\mathcal{L}$, to the lowest $Q^{2}$ order, under the identifications

$$
\begin{aligned}
\frac{f_{\pi q q}^{2}}{M_{\pi}^{2}} & =\frac{g_{\pi q q}^{2}}{4 M_{q}^{2}} \\
\frac{\left(f_{\pi N N}^{2}\right)_{Q^{2}=M_{\pi}^{2}}^{2}}{M_{\pi}^{2}} & =\frac{\left(g_{\pi N N}^{2}\right)_{Q^{2}=M_{\pi}^{2}}}{4 M_{N}^{2}} .
\end{aligned}
$$

If we now substitute these relations in Eq. (22) we get

$$
\left(f_{\pi N N}^{2}\right)_{Q^{2}=M_{\pi}^{2}}=f_{\pi q q}^{2} \frac{25}{9} e^{\frac{M_{\pi}^{2} b^{2}}{2}} .
$$

The corresponding constant $f_{c h}$ is consistently defined as

$$
f_{c h}=\left(\frac{\Lambda^{2}-M_{\pi}^{2}}{\Lambda^{2}}\right)^{1 / 2} f_{\pi q q}=\lim _{M_{\pi} \rightarrow 0} f_{\pi q q} .
$$

\section{Coupling constants and form factor values}

From a standard experimental value $\left(f_{\pi N N}^{2}\right)_{Q^{2}=M_{\pi}^{2}} / 4 \pi \simeq 0.079$ (see [1] and references therein) or $\left(g_{\pi N N}^{2}\right)_{Q^{2}=M_{\pi}^{2}} / 4 \pi \simeq 14.6$ we fit the pion-quark-quark coupling constant

$$
f_{\pi q q}^{2} / 4 \pi=0.027
$$

or

$$
g_{\pi q q}^{2} / 4 \pi=0.55
$$

and

$$
\left|g_{c h}\right| \equiv\left|f_{c h}\right| \frac{2 M_{q}}{M_{\pi}}=2.6 .
$$

Let us emphasize that this value for $g_{c h}$ differs less than a $10 \%$ from the one obtained via QCD sum rules (QCDSR) $\left(g_{c h}\right)_{\mathrm{QCDSR}} \simeq 2.83[12]$.

Regarding $\Lambda_{\pi N N}$, the cutoff parameter, its range of values can be estimated. In Ref. [13] a fit to data was attained from a gaussian form factor $e^{Q^{2} / \Lambda_{\mathrm{HM}}^{2}}$ with $\Lambda_{\mathrm{HM}}$ varying from 2.6 to $4.2 \mathrm{fm}^{-1}$. This form factor is normalized at $Q^{2}=0$. By requiring its low $Q^{2}$ behavior 
$\left(1+Q^{2} / \Lambda_{\mathrm{HM}}^{2}\right)$, to be the same than that of our form factor normalized at $Q^{2}=0$ in Eq. (20), $\left(1+Q^{2} / 2 \Lambda_{\pi N N}^{2}\right)$, we get $\Lambda_{\mathrm{HM}}^{2}=2 \Lambda_{\pi N N}^{2}$. Hence the resulting range for $\Lambda_{\pi N N}$ is

$$
\Lambda_{\pi N N}=1.84-2.97 \mathrm{fm}^{-1} \text {. }
$$

From Eqs. (19) and (20) this range can be translated in an interval of values for the coupling at $Q^{2}=0$ :

$$
\begin{aligned}
& \frac{\left(f_{\pi N N}^{2}\right)_{Q^{2}=0}}{4 \pi}=0.068-0.075, \\
& \frac{\left(g_{\pi N N}^{2}\right)_{Q^{2}=0}}{4 \pi}=12.5-13.8 .
\end{aligned}
$$

These values are in perfect agreement with the phenomenological analysis done in Ref. [14] (let us comment that for the form factor used in this reference the range of values for the cutoff parameter is the same as for $\left.\Lambda_{\mathrm{HM}}\right)$. The preferred value in Ref. [14] is $\left(f_{\pi N N}^{2}\right)_{Q^{2}=0} / 4 \pi \simeq$ 0.073 which corresponds to $\left(g_{\pi N N}^{2}\right)_{Q^{2}=0} / 4 \pi \simeq 13.5$. It is worthwhile to point out that this corresponds quite approximately to the $M_{\pi} \rightarrow 0$ limit of our expression $\left(f_{\pi N N}^{2}\right)_{Q^{2}=M_{\pi}^{2}}=$ $f_{\pi q q}^{2}(25 / 9) e^{\frac{M_{\pi}^{2} b^{2}}{2}}$, i.e.,

$$
\frac{\left(f_{\pi N N}^{2}\right)_{Q^{2}=M_{\pi}^{2} \rightarrow 0}}{4 \pi}=\frac{f_{c h}^{2}}{4 \pi} \frac{25}{9}=0.072
$$

indicating the quite approximate Goldstone boson character of the pion.

\section{D. $\sigma \mathbf{N N}$}

By proceeding in exactly the same way for the $\sigma$ exchange we obtain from Eqs. (11), (13) and (16) at the quark level

$$
\left(V_{q}\right)_{\mathrm{OSE}}^{N N \rightarrow N N}(R \rightarrow \infty)=-\frac{g_{\sigma q q}^{2}}{4 \pi} 9 e^{\frac{M_{\sigma}^{2} b^{2}}{2}} \frac{e^{-M_{\sigma} R}}{R} .
$$

On the other hand from the hadronic lagrangian

$$
\mathcal{L}_{\sigma N N}=-\left(g_{\sigma N N}\right)_{Q^{2}=M_{\sigma}^{2}} \bar{N} \mathbb{1}_{S U(2)} \sigma N,
$$

with a vertex form factor

$$
G_{\sigma N N}\left(Q^{2}\right)=\left(\frac{\Lambda_{\sigma N N}^{2}-M_{\sigma}^{2}}{\Lambda_{\sigma N N}^{2}-Q^{2}}\right)^{\frac{1}{2}}
$$

we get

$$
\left(\mathcal{V}_{B}\right)_{\mathrm{OSE}}^{N N \rightarrow N N}(R \rightarrow \infty)=-\frac{\left(g_{\sigma N N}^{2}\right)_{Q^{2}=M_{\sigma}^{2}}}{4 \pi} \frac{e^{-M_{\sigma} R}}{R}
$$

From their comparison

$$
\left(g_{\sigma N N}^{2}\right)_{Q^{2}=M_{\sigma}^{2}}=9 g_{\sigma q q}^{2} e^{\frac{M_{\sigma}^{2} b^{2}}{2}} .
$$

Let us note that once the value of $g_{\pi q q}$ has been fitted our model predicts the value of $g_{\sigma q q}$ through

$$
\frac{g_{\sigma q q}^{2}}{g_{\pi q q}^{2}}=\frac{\Lambda^{2}-M_{\pi}^{2}}{\Lambda^{2}-M_{\sigma}^{2}} .
$$


Equivalently

$$
\frac{\left(g_{\sigma N N}^{2}\right)_{Q^{2}=M_{\sigma}^{2}}}{\left(g_{\pi N N}^{2}\right)_{Q^{2}=M_{\pi}^{2}}}=\frac{9}{25} \frac{\Lambda^{2}-M_{\pi}^{2}}{\Lambda^{2}-M_{\sigma}^{2}} e^{\frac{\left(M_{\sigma}^{2}-M_{\pi}^{2}\right) b^{2}}{2}} .
$$

\section{Coupling constant and form factor values}

From Eq. (42) we predict

$$
\frac{\left(g_{\sigma N N}^{2}\right)_{Q^{2}=M_{\sigma}^{2}}}{4 \pi}=68.2
$$

and from Eq. (40)

$$
\frac{g_{\sigma q q}^{2}}{4 \pi}=1.59
$$

As mentioned above our asymptotic comparison does not give any information on the cutoff parameter $\Lambda_{\sigma N N}$. Nonetheless we can combine our result for the coupling constant with the value of the coupling at $Q^{2}=0$ provided by QCDSR to get an insight into it. From Ref. [15]

$$
\frac{\left(g_{\sigma N N}\right)_{Q^{2}=0}}{g_{q}^{\sigma}}=3.9 \pm 1.0 .
$$

If we tentatively identify $g_{q}^{\sigma}$ with our $g_{\sigma q q}(=4.47)$ we get

$$
\frac{\left(g_{\sigma N N}^{2}\right)_{Q^{2}=0}}{4 \pi}=17.4 \pm 4.5
$$

and using the relation $\left(g_{\sigma N N}^{2}\right)_{Q^{2}=0}=\left(g_{\sigma N N}^{2}\right)_{Q^{2}=M_{\sigma}^{2}} G_{\sigma N N}^{2}\left(Q^{2}=0\right)$ we extract

$$
\Lambda_{\sigma N N}=\left(\frac{M_{\sigma}^{2}}{1-\frac{\left(g_{\sigma N N}^{2}\right)_{Q^{2}=0}}{\left(g_{\sigma N N}^{2}\right)_{Q^{2}=M_{\sigma}^{2}}}}\right)^{\frac{1}{2}}=3.97 \pm 0.18 \mathrm{fm}^{-1} .
$$

It is again interesting to consider the limit $M_{\sigma} \rightarrow 0$ of Eq. (40)

$$
\frac{\left(g_{\sigma N N}^{2}\right)_{Q^{2}=M_{\sigma}^{2} \rightarrow 0}}{4 \pi}=\frac{9 g_{c h}^{2}}{4 \pi}=4.8
$$

or

$$
\frac{\left(g_{\sigma N N}^{2}\right)_{Q^{2}=M_{\sigma}^{2} \rightarrow 0}}{g_{\mathrm{ch}}^{2}}=9
$$

and compare it to the interval of values of the coupling at $Q^{2}=0$ from Eq. (46)). As can be seen the $M_{\sigma} \rightarrow 0$ value from Eq. (48) is out of this interval. This might be interpreted as reflecting the non-Goldstone boson nature of the $\sigma$. 


\section{E. $\pi \mathbf{N} \Delta$}

Strictly speaking our procedure to extract the couplings only makes sense when the lowest order expansion in $Q^{2}$ we follow is simultaneously valid at the baryonic level $\left(E_{B} \simeq M_{B}\right)$ and at the quark level $\left(E_{q} \simeq M_{q}\right)$. We do not expect this to be true for light-quark baryons in general since the quarks can move relativistically inside them. However the structure of the ground states, the $N$ (and $\Lambda, \Sigma$, and $\Xi$ when considering $S U(3)$ ), is well described by non-relativistic constituent quark models through the effective parameters in the quarkquark potential. Then we expect our procedure to make sense for them. Regarding other baryon states like $\Delta$ and $N(1440)$ one should be more cautious as we illustrate next.

In order to extract the $\pi N \Delta$ coupling constant we consider the $N N \rightarrow N \Delta$ interaction. According to the harmonic oscillator model we are using the spatial wave function of $\Delta$ has exactly the same structure than the $N$ one. However the real $\Delta$ differs from the $N$. To implement the bigger size for $\Delta$ predicted by non-relativistic spectroscopic models we shall consider the possibility of a slightly different value for the size parameter. Thus the $\Delta$ spatial wave function we shall use is

$$
\left(\Phi_{\Delta}\right)_{\text {spatial }}(4,5,6 ;+\vec{R} / 2)=\prod_{i=4}^{6}\left(\frac{1}{\pi b_{\Delta}^{2}}\right)^{\frac{3}{4}} \exp \left[-\left(\vec{r}_{i}-\vec{R} / 2\right)^{2} / 2 b_{\Delta}^{2}\right]
$$

with a baryon size parameter, $b_{\Delta}$. One should also keep in mind that the mass of the $\Delta$ is a $30 \%$ bigger than the mass of the $N$. In our harmonic oscillator quark model this means that the quarks in the $\Delta$ have more potential and kinetic energy (virial theorem) than the quarks in the $N$. According to our comments above this could give rise to corrections in the expression of the static potential at the quark level. Moreover due to the $\Delta-N$ mass difference the positions of the baryons in the initial and final states should not be the same. Therefore we should not expect an accurate prediction for the coupling constant in this case. To derive such prediction we first calculate at the quark level, from Eqs. (13), (16) and (150)

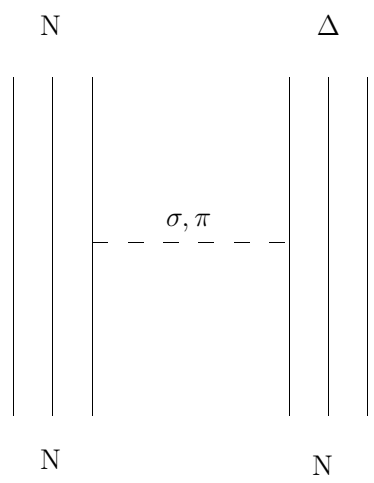

FIG. 3: Asymptotic $N N \rightarrow N \Delta$ OPE or OSE interactions at quark level.

(we use for easiness the pseudovector form of the coupling) the asymptotic $N N \rightarrow N \Delta$ pion exchange static central potential, Fig. 3, It is

$$
\left(V_{q}\right)_{\mathrm{OPE}}^{N N \rightarrow N \Delta}(R \rightarrow \infty)=\frac{f_{\pi q q}^{2}}{4 \pi}\left[\frac{160}{9 \sqrt{2}} 2 \sqrt{2} \frac{b_{\mu}^{6} b_{+}^{3} b_{e}^{3}}{\left(b^{15} b_{\Delta}^{9}\right)^{\frac{1}{2}}} e^{\frac{M_{\pi}^{2} b_{e}^{2}}{2}}\right] \frac{1}{3} \frac{e^{-M_{\pi} R}}{R},
$$


where the factor $160 / 9 \sqrt{2}$ corresponds to $\left\langle N \Delta\left|\left(\vec{\sigma}_{3} \cdot \vec{\sigma}_{6}\right)\left(\vec{\tau}_{3} \cdot \vec{\tau}_{6}\right)\right| N N\right\rangle$ for total spin and isospin equal to 1 , multiplied by 9 the number of equivalent diagrams, and $b_{\mu}, b_{+}$and $b_{e}$ are defined as

$$
\begin{aligned}
\frac{1}{b_{\mu}^{2}} & =\frac{1}{2 b^{2}}+\frac{1}{2 b_{\Delta}^{2}} \\
\frac{1}{b_{ \pm}^{2}} & = \pm \frac{1}{b^{2}}+\frac{1}{b_{\mu}^{2}} \\
\frac{1}{b_{e}^{2}} & =\frac{1}{2 b_{+}^{2}}-\frac{b_{+}^{2}}{2 b_{-}^{4}} .
\end{aligned}
$$

Note that when $b_{\Delta}=b$ we also have $b_{e}=b_{\mu}=\sqrt{2} b_{+}=b$ hence reproducing, except for the spin-isospin factor, the $N N$ result.

At the baryonic level we have the lagrangians $\mathcal{L}_{\pi N N}^{P V}$ as given by Eq. (25) and

$$
\mathcal{L}_{\pi N \Delta}^{P V}=-\frac{\left(f_{\pi N \Delta}\right)_{Q^{2}=M_{\pi}^{2}}}{M_{\pi}} \bar{N} \vec{T} \partial \vec{\pi} \Delta
$$

with a form factor $G_{\pi N \Delta}\left(Q^{2}\right)$ which we shall choose as,

$$
G_{\pi N \Delta}\left(Q^{2}\right) \equiv\left(\frac{\Lambda_{\pi N \Delta}^{2}-M_{\pi}^{2}}{\Lambda_{\pi N \Delta}^{2}-Q^{2}}\right)^{\frac{1}{2}}
$$

In Eq. (153) $\Delta$ corresponds to a Rarita-Schwinger $3 / 2$ spinor field and $\vec{T}$ is the isospin nucleondelta transition operator. The corresponding pion exchange static central potential behaves asymptotically as

$$
\left(\mathcal{V}_{B}\right)_{\mathrm{OPE}}^{N N \rightarrow N \Delta}(R \rightarrow \infty)=\frac{\left(f_{\pi N N}\right)_{Q^{2}=M_{\pi}^{2}}\left(f_{\pi N \Delta}\right)_{Q^{2}=M_{\pi}^{2}}}{4 \pi}\left[\left\langle\left(\vec{\sigma}_{N} \cdot \vec{S}\right)\left(\vec{\tau}_{N} \cdot \vec{T}\right)\right\rangle\right] \frac{1}{3} \frac{e^{-M_{\pi} R}}{R}
$$

where $\vec{S}$ is the spin nucleon-delta transition operator and $\left\langle\left(\vec{\sigma}_{N} \cdot \vec{S}\right)\left(\vec{\tau}_{N} \cdot \vec{T}\right)\right\rangle=8 / 3$ for total spin and isospin equal to 1 . Thus we identify,

$$
\frac{\left(f_{\pi N N}\right)_{Q^{2}=M_{\pi}^{2}}\left(f_{\pi N \Delta}\right)_{Q^{2}=M_{\pi}^{2}}}{4 \pi}=\frac{f_{\pi q q}^{2}}{4 \pi}\left[\frac{20}{3 \sqrt{2}} 2 \sqrt{2} \frac{b_{\mu}^{6} b_{+}^{3} b_{e}^{3}}{\left(b^{15} b_{\Delta}^{9}\right)^{\frac{1}{2}}} e^{\frac{M_{\pi}^{2} b_{e}^{2}}{2}}\right] .
$$

By using Eq. (28) we get,

$$
\frac{\left(f_{\pi N \Delta}\right)_{Q^{2}=M_{\pi}^{2}}}{\left(f_{\pi N N}\right)_{Q^{2}=M_{\pi}^{2}}}=\left[\frac{6 \sqrt{2}}{5} 2 \sqrt{2} \frac{b_{\mu}^{6} b_{+}^{3} b_{e}^{3}}{\left(b^{15} b_{\Delta}^{9}\right)^{\frac{1}{2}}} e^{\frac{M_{\pi}^{2}\left(b_{e}^{2}-b^{2}\right)}{2}}\right]
$$

so that for $b_{\Delta}=b$ one obtains the usual spin-isospin relation $\left(f_{\pi N \Delta}\right)_{Q^{2}=M_{\pi}^{2}} /\left(f_{\pi N N}\right)_{Q^{2}=M_{\pi}^{2}}=$ $6 \sqrt{2} / 5$.

For $b_{\Delta}$ in the interval $[b, 1.2 b]$ we predict $\left(f_{\pi N \Delta}^{2}\right)_{Q^{2}=M_{\pi}^{2}} / 4 \pi=[0.23,0.20]$ to be compared to $\left(f_{\pi N \Delta}^{2}\right)_{Q^{2}=M_{\pi}^{2}} / 4 \pi \simeq 0.37$, estimated from the $\Delta$ decay to $N \pi$. This discrepancy seems to confirm our initial expectations. If instead $f_{\pi q q}$ we had written an effective $\left(f_{\pi q q}\right)_{N \Delta}$ as a manner to take into account the $\Delta-N$ mass difference effect then the needed value 
to reproduce the experimental number would have been $\left(f_{\pi q q}\right)_{N \Delta} / f_{\pi q q}=[1.28,1.37]$ for $b_{\Delta} \in[b, 1.2 b]$, i.e. $\left(f_{\pi q q}\right)_{N \Delta}$ should be a $30 \%$ bigger than $f_{\pi q q}$.

For nucleon resonances in general and in particular for $N^{*}(1440)$ we expect the calculation of the coupling constants to be much more uncertain. As a matter of fact the importance of relativistic corrections in the description of the structure and decay of $N^{*}(1440)$ in terms of quarks have been emphasized for a long time in the literature (see for instance [16]). Furthermore the nature of the $N^{*}(1440)$ may involve more than a simple $3 q$ structure and the coupling of $q \bar{q}$ pairs to the meson structure can be relevant. Therefore the calculation of $\pi N N^{*}(1440)$ and $\sigma N N^{*}(1440)$ coupling constants carried out in a preceding paper within the same framework [17] should be considered too simplistic. A less approximative calculation for the pion case (involving also $\pi N \Delta, \pi N \Delta(1600)$ and $\pi N N(1535)$ ) has been carried out in reference [18] with Poincaré covariant constituent quark models with instant, point and front forms of relativistic kinematics; from the persistent deviation from data of the calculated results the authors suggest the presence of sizable $q q q q \bar{q}$ components in the baryon wave functions.

\section{LIGHT AND STRANGE QUARK SECTOR: $\mathrm{SU}(3) \otimes \mathrm{SU}(3)$.}

The generalization of the chiral lagrangian to $\mathrm{SU}(3) \otimes \mathrm{SU}(3)$ is straightforward. It is expressed as

$$
\tilde{\mathcal{L}}_{c h}=-\tilde{g}_{c h} \bar{q}\left(\sum_{a=0}^{8} \sigma_{a} \lambda_{a}+i \sum_{a=0}^{8} \gamma_{5} \pi_{a} \lambda_{a}\right) q,
$$

where $q$ has components $u, d$ and $s, \sigma_{0}$ and $\pi_{0}$ stand for the scalar and pseudoscalar meson singlets whereas $\sigma_{i}$ and $\pi_{i}(i=1 \ldots 8)$ are the scalar and pseudoscalar meson octets.

In order to derive a potential involving the exchange of $\sigma$, the $\mathrm{SU}(2)$ singlet, we shall assume the ideal mixing

$$
\begin{aligned}
& \sigma_{0}=\sqrt{2 / 3} \sigma+\sqrt{1 / 3}(s \bar{s}) \\
& \sigma_{8}=\sqrt{1 / 3} \sigma-\sqrt{2 / 3}(s \bar{s}) .
\end{aligned}
$$

When substituting these expressions in Eq. (58) the piece containing the $\sigma$ and the $\vec{\pi}$ read,

$$
\tilde{\mathcal{L}}_{c h(\pi, \sigma)}=-\tilde{g}_{c h} \bar{q}\left[\sigma\left(\sqrt{2 / 3} \lambda_{0}+\sqrt{1 / 3} \lambda_{8}\right)+i \gamma_{5} \vec{\tau} \vec{\pi}\right] q
$$

where $\lambda_{0} \equiv \sqrt{2 / 3} \mathbb{1}_{\mathrm{SU}(3)}$ and $\lambda_{8} \equiv \sqrt{3} Y$, being $Y$ the hypercharge. It is then clear that for $q=u, d(Y u=1 / 3=Y d)$ one formally recovers the $\mathrm{SU}(2) \otimes \mathrm{SU}(2)$ lagrangian: $\mathbb{1}_{\mathrm{SU}(2)} \equiv$ $2 / 3 \mathbb{1}_{\mathrm{SU}(3)}+Y$.

Again we take into account SCSB through a vertex form factor $\tilde{F}\left(Q^{2}\right)$

$$
\tilde{F}\left(Q^{2}\right)=\left(\frac{\tilde{\Lambda}^{2}}{\tilde{\Lambda}^{2}-Q^{2}}\right)^{1 / 2}
$$

Note also that the SCSB relation, $M_{\sigma}^{2}-M_{\pi}^{2}=4 M_{q}^{2}$, is preserved since it is derived for a non-strange $\sigma[11]$. 


\section{A. Parameters}

One should realize that the values of the couplings $\tilde{g}_{c h} \tilde{F}\left(Q^{2}\right)$ (or equivalently the on-shell couplings $\tilde{g}_{\pi q q}, \tilde{g}_{\sigma q q}$ and the cutoff parameter $\left.\tilde{\Lambda}\right)$ and the size parameter $\tilde{b}$ in $\mathrm{SU}(3) \otimes \mathrm{SU}(3)$ need not be the same as in $\mathrm{SU}(2) \otimes \mathrm{SU}(2)$. This is easily understandable by thinking for instance of the extra contribution to the $N N$ interaction coming from $\eta, \eta^{\prime}$ and $a_{0}$ in $\mathrm{SU}(3) \otimes \mathrm{SU}(3)$. This contribution is taken into account in an effective manner in $\mathrm{SU}(2) \otimes \mathrm{SU}(2)$, where no $\eta, \eta^{\prime}$, and $a_{0}$ are present, through the fitted values of $g_{\pi q q}, g_{\sigma q q}$, $\Lambda$ and $b$. Fortunately we can correlate the variations of $b$ and $\Lambda$ (or $\tilde{b}$ and $\tilde{\Lambda}$ ) through the relation Eq. (42),

$$
\frac{\left(g_{\sigma N N}^{2}\right)_{Q^{2}=M_{\sigma}^{2}}}{\left(g_{\pi N N}^{2}\right)_{Q^{2}=M_{\pi}^{2}}}=\frac{9}{25} \frac{\Lambda^{2}-M_{\pi}^{2}}{\Lambda^{2}-M_{\sigma}^{2}} e^{\frac{\left(M_{\sigma}^{2}-M_{\pi}^{2}\right) b^{2}}{2}}=\frac{9}{25} \frac{\tilde{\Lambda}^{2}-M_{\pi}^{2}}{\tilde{\Lambda}^{2}-M_{\sigma}^{2}} e^{\frac{\left(M_{\sigma}^{2}-M_{\pi}^{2}\right) \tilde{b}^{2}}{2}} .
$$

Then from the selected experimental value $\left(g_{\pi N N}^{2}\right)_{Q^{2}=M_{\pi}^{2}} / 4 \pi \simeq 14.6$ and from our prediction $\left(g_{\sigma N N}^{2}\right)_{Q^{2}=M_{\sigma}^{2}} / 4 \pi=68.2$ we get, for a typical value of $\tilde{\Lambda} \simeq 5.2 \mathrm{fm}^{-1}(\simeq 1.0 \mathrm{GeV})$ (note that it has to be higher than any mass of the scalar or pseudoscalar meson octets) an harmonic oscillator parameter $\tilde{b} \simeq 0.6 \mathrm{fm}$. Then from equivalent relations to Eqs. (23) and (40) we get

$$
\begin{gathered}
\frac{\tilde{g}_{\pi q q}^{2}}{4 \pi}=0.54 \\
\frac{\tilde{g}_{\sigma q q}^{2}}{4 \pi}=0.93
\end{gathered}
$$

and

$$
\tilde{g}_{c h}=g_{c h} \text {. }
$$

For the sake of completeness we give the $B_{8}$ spatial wave function in the $\mathrm{SU}(3)_{\text {flavor }}$ limit,

$$
\left(\Phi_{B_{8}}\right)_{\text {spatial }}(1,2,3 ;+\vec{R} / 2)=\prod_{i=1}^{3}\left(\frac{1}{\pi \tilde{b}^{2}}\right)^{\frac{3}{4}} \exp \left[-\left(\vec{r}_{i}-\vec{R} / 2\right)^{2} / 2 \tilde{b}^{2}\right] \text {. }
$$

\section{B. $\sigma \mathbf{B}_{8} \mathbf{B}_{8}$}

According to our preceding discussions the $\sigma q q$ lagrangian will be written as

$$
\tilde{\mathcal{L}}_{\sigma q q}=-\tilde{g}_{\sigma q q} \bar{q} \mathbb{1}_{\mathrm{SU}(2)} \sigma q
$$

with a vertex form factor

$$
\tilde{F}_{\sigma q q}\left(Q^{2}\right) \equiv\left(\frac{\tilde{\Lambda}^{2}-M_{\sigma}^{2}}{\tilde{\Lambda}^{2}-Q^{2}}\right)^{1 / 2}
$$

From this lagrangian it is clear that the only difference when calculating the asymptotic potential at the quark level for the several $B_{8} B_{8}$ 's has to do with the number of the pairs of light quarks $(u, d)$ in them allowing for the exchange of the $\sigma$, i.e., with the number of 


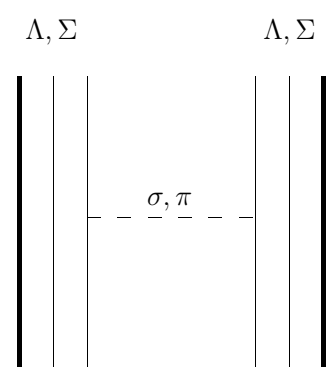

$\Lambda, \Sigma$

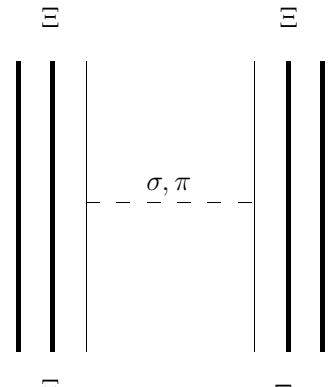

$\Xi$

FIG. 4: Asymptotic $\Lambda \Lambda, \Sigma \Sigma$, and $\Xi \Xi$ OPE and OSE interactions at quark level. Thin lines stand for light $(u, d)$ quarks and thick lines for strange $(s)$ quarks.

equivalent diagrams, Figs. 1 and 4. This number is 9 for $N N, 4$ for $\Lambda \Lambda$ and $\Sigma \Sigma$, and 1 for $\Xi \Xi$. Thus

$$
\begin{aligned}
& \left(\tilde{V}_{q}\right)_{\mathrm{OSE}}^{\Lambda \Lambda \rightarrow \Lambda \Lambda}(R \rightarrow \infty)=\left(\tilde{V}_{q}\right)_{\mathrm{OSE}}^{\Sigma \Sigma \rightarrow \Sigma \Sigma}(R \rightarrow \infty)=\frac{4}{9}\left(\tilde{V}_{q}\right)_{\mathrm{OSE}}^{N N \rightarrow N N}(R \rightarrow \infty) \\
& \left(\tilde{V}_{q}\right)_{\mathrm{OSE}}^{\Xi \Xi \exists \Xi}(R \rightarrow \infty)=\frac{1}{9}\left(\tilde{V}_{q}\right)_{\mathrm{OSE}}^{N N \rightarrow N N}(R \rightarrow \infty) .
\end{aligned}
$$

On the other hand at the baryonic level we shall write the lagrangian as

$$
\mathcal{L}_{\sigma B_{8} B_{8}} \equiv-\left(g_{\sigma B_{8} B_{8}}\right)_{Q^{2}=M_{\sigma}^{2}} \bar{B}_{8} \sigma B_{8}
$$

with a vertex form factor

$$
G_{\sigma B_{8} B_{8}}\left(Q^{2}\right)=\left(\frac{\Lambda_{\sigma B_{8} B_{8}}^{2}-M_{\sigma}^{2}}{\Lambda_{\sigma B_{8} B_{8}}^{2}-Q^{2}}\right)^{1 / 2} .
$$

so that $G_{\sigma B_{8} B_{8}}\left(Q^{2}=M_{\sigma}^{2}\right)=1$ and where the $\left(g_{\sigma B_{8} B_{8}}\right)_{Q^{2}=M_{\sigma}^{2}}$ are expressed in conventional notation

$$
\begin{aligned}
&\left(g_{\sigma N N}\right)_{Q^{2}=M_{\sigma}^{2}} \equiv \sqrt{2 / 3} g_{s, 1}+1 / 3 g_{s,+}\left(4 \alpha_{s}-1\right) \\
&\left(g_{\sigma \Lambda \Lambda}\right)_{Q^{2}=M_{\sigma}^{2}} \equiv \sqrt{2 / 3} g_{s, 1}-2 / 3 g_{s,+}\left(1-\alpha_{s}\right) \\
&\left(g_{\sigma \Sigma \Sigma}\right)_{Q^{2}=M_{\sigma}^{2}} \equiv \sqrt{2 / 3} g_{s, 1}+2 / 3 g_{s,+}\left(1-\alpha_{s}\right) \\
&\left(g_{\sigma \Xi \Xi}\right)_{Q^{2}=M_{\sigma}^{2}} \equiv \sqrt{2 / 3} g_{s, 1}-1 / 3 g_{s,+}\left(1+2 \alpha_{s}\right)
\end{aligned}
$$

being $g_{s, 1} \equiv\left(g_{\sigma_{0} B_{8} B_{8}}\right)_{Q^{2}=M_{\sigma}^{2}}$ the scalar SU(3) singlet coupling constant, $g_{s,+} \equiv g_{s, D}+g_{s, F}$ the sum of the $D$ (symmetric) and the $F$ (antisymmetric) scalar coupling constants in $\mathrm{SU}(3)$ and $\alpha_{s} \equiv g_{s, F} / g_{s,+}$ the $F /(F+D)$ ratio of the scalar octet.

From this baryonic lagrangian the following relations between the asymptotic potentials 
come out

$$
\begin{aligned}
& \left(\mathcal{V}_{B}\right)_{\mathrm{OSE}}^{\Lambda \Lambda \rightarrow \Lambda \Lambda}(R \rightarrow \infty)=\frac{\left(g_{\sigma \Lambda \Lambda}^{2}\right)_{Q^{2}=M_{\sigma}^{2}}}{\left(g_{\sigma N N}^{2}\right)_{Q^{2}=M_{\sigma}^{2}}}\left(\mathcal{V}_{B}\right)_{\mathrm{OSE}}^{N N \rightarrow N N}(R \rightarrow \infty) \\
& \left(\mathcal{V}_{B}\right)_{\mathrm{OSE}}^{\Sigma \Sigma \rightarrow \Sigma \Sigma}(R \rightarrow \infty)=\frac{\left(g_{\sigma \Sigma \Sigma}^{2}\right)_{Q^{2}=M_{\sigma}^{2}}}{\left(g_{\sigma N N}^{2}\right)_{Q^{2}=M_{\sigma}^{2}}}\left(\mathcal{V}_{B}\right)_{\mathrm{OSE}}^{N N \rightarrow N N}(R \rightarrow \infty) \\
& \left(\mathcal{V}_{B}\right)_{\mathrm{OSE}}^{\Xi \Xi \rightarrow \Xi \Xi}(R \rightarrow \infty)=\frac{\left(g_{\sigma \Xi \Xi}^{2}\right)_{Q^{2}=M_{\sigma}^{2}}}{\left(g_{\sigma N N}^{2}\right)_{Q^{2}=M_{\sigma}^{2}}}\left(\mathcal{V}_{B}\right)_{\mathrm{OSE}}^{N N \rightarrow N N}(R \rightarrow \infty)
\end{aligned}
$$

From the comparison of the asymptotic potentials at the quark and baryon levels we immediately get relations between the coupling constants

$$
\begin{aligned}
\frac{\left(g_{\sigma \Lambda \Lambda}^{2}\right)_{Q^{2}=M_{\sigma}^{2}}}{\left(g_{\sigma N N}^{2}\right)_{Q^{2}=M_{\sigma}^{2}}}=\frac{\left(g_{\sigma \Sigma \Sigma}^{2}\right)_{Q^{2}=M_{\sigma}^{2}}}{\left(g_{\sigma N N}^{2}\right)_{Q^{2}=M_{\sigma}^{2}}}=\frac{4}{9} \\
\frac{\left(g_{\sigma \Xi \Xi}^{2}\right)_{Q^{2}=M_{\sigma}^{2}}}{\left(g_{\sigma N N}^{2}\right)_{Q^{2}=M_{\sigma}^{2}}}=\frac{1}{9} .
\end{aligned}
$$

We should emphasize that these ratios are preserved in the limit $M_{\sigma} \rightarrow 0$, i.e.,

$$
\begin{aligned}
& \frac{\left(g_{\sigma \Lambda \Lambda}^{2}\right)_{Q^{2}=M_{\sigma}^{2} \rightarrow 0}}{\left(g_{\sigma N N}^{2}\right)_{Q^{2}=M_{\sigma}^{2} \rightarrow 0}}=\frac{\left(g_{\sigma \Sigma \Sigma}^{2}\right)_{Q^{2}=M_{\sigma}^{2} \rightarrow 0}}{\left(g_{\sigma N N}^{2}\right)_{Q^{2}=M_{\sigma}^{2} \rightarrow 0}}=\frac{4}{9} \\
& \frac{\left(g_{\sigma \Xi \Xi}^{2}\right)_{Q^{2}=M_{\sigma}^{2} \rightarrow 0}}{\left(g_{\sigma N N}^{2}\right)_{Q^{2}=M_{\sigma}^{2} \rightarrow 0}}=\frac{1}{9} .
\end{aligned}
$$

It is interesting to compare these $M_{\sigma} \rightarrow 0$ ratios with the average QCDSR predictions at $Q^{2}=0$ in the $\mathrm{SU}(3)$ limit. From Ref. [19] we take

$$
\begin{aligned}
& \frac{\left(g_{\sigma \Lambda \Lambda}^{2}\right)_{Q^{2}=0}}{\left(g_{\sigma N N}^{2}\right)_{Q^{2}=0}}=(0.43)^{2}=0.19 \\
& \frac{\left(g_{\sigma \Sigma \Sigma}^{2}\right)_{Q^{2}=0}}{\left(g_{\sigma N N}^{2}\right)_{Q^{2}=0}}=(0.91)^{2}=0.83 \\
& \frac{\left(g_{\sigma \Xi \Xi}^{2}\right)_{Q^{2}=0}}{\left(g_{\sigma N N}^{2}\right)_{Q^{2}=0}}=(0.08)^{2}=0.006 .
\end{aligned}
$$

As can be checked our ratios differ by a factor $2(1 / 2)$ for $\Lambda-N$ and $\Sigma-N(\Xi-N)$ from the QCDSR ones. We may interpret this again as a reflection of the non-Goldstone character of the $\sigma$ meson.

\section{Coupling constants and form factors values}

From Eq. (74) and from the calculated value $\left(g_{\sigma N N}^{2}\right)_{Q^{2}=M_{\sigma}^{2}} / 4 \pi=68.2$ we predict the coupling constants

$$
\begin{aligned}
& \frac{\left(g_{\sigma \Lambda \Lambda}^{2}\right)_{Q^{2}=M_{\sigma}^{2}}}{4 \pi}=\frac{\left(g_{\sigma \Sigma \Sigma}^{2}\right)_{Q^{2}=M_{\sigma}^{2}}}{4 \pi}=30.3 \\
& \frac{\left(g_{\sigma \Xi \Xi}^{2}\right)_{Q^{2}=M_{\sigma}^{2}}}{4 \pi}=7.6 .
\end{aligned}
$$


Concerning the $F /(F+D)$ ratio we have from Eq. (174) $\left(g_{\sigma \Lambda \Lambda}\right)_{Q^{2}=M_{\sigma}^{2}}=\left(g_{\sigma \Sigma \Sigma}\right)_{Q^{2}=M_{\sigma}^{2}}$ what implies from Eq. (72)

$$
\alpha_{s}=1
$$

or

$$
g_{S, D}=0 .
$$

Regarding $g_{s, 1}$ and $g_{S,+}$ we can use the fact that from the ideal mixing we have assumed $g_{(s \bar{s}) N N}=-1 / \sqrt{3} g_{s, 1}+\sqrt{2} g_{s, F}-\sqrt{2} / 3 g_{s, D}=0$. Then from Eq. (79) we immediately obtain $g_{s,+}=1 / \sqrt{6} g_{s, 1}$. If we substitute this relation and Eq. (78) in the first expression of Eq. (72) we get $\left(g_{\sigma N N}\right)_{Q^{2}=M_{\sigma}^{2}}=3 g_{s,+}$ from where

$$
\begin{aligned}
& \frac{g_{s,+}^{2}}{4 \pi}=7.6 \\
& \frac{g_{s, 1}^{2}}{4 \pi}=45.5
\end{aligned}
$$

satisfying

$$
\frac{g_{s, 1}^{2}}{g_{s,+}^{2}}=6 .
$$

With respect to the cutoff parameters $\Lambda_{\sigma B_{8} B_{8}}$ we can tentatively use our on-shell couplings ratios, Eq. (74), altogether with the QCDSR ones at $Q^{2}=0$ detailed above, Eq. (76)), to establish a range of variation for them. Explicitly we can write

$$
\left(\frac{\left(g_{\sigma B_{8} B_{8}}^{2}\right)_{Q^{2}=0}}{\left(g_{\sigma N N}^{2}\right)_{Q^{2}=0}}\right)=\left(\frac{\left(g_{\sigma B_{8} B_{8}}^{2}\right)_{Q^{2}=M_{\sigma}^{2}}}{\left(g_{\sigma N N}^{2}\right)_{Q^{2}=M_{\sigma}^{2}}}\right) \frac{\left(\frac{\Lambda_{\sigma B_{8} B_{8}}^{2}-M_{\sigma}^{2}}{\Lambda_{\sigma B_{8} B_{8}}^{2}}\right)}{\left(\frac{\Lambda_{\sigma N N}^{2}-M_{\sigma}^{2}}{\Lambda_{\sigma N N}^{2}}\right)}
$$

or equivalently

$$
\Lambda_{\sigma B_{8} B_{8}}=\Lambda_{\sigma N N}\left(\frac{M_{\sigma}^{2}}{\Lambda_{\sigma N N}^{2}\left(1-x_{B_{8}}\right)+x_{B_{8}} M_{\sigma}^{2}}\right)^{\frac{1}{2}}
$$

where

$$
x_{B_{8}} \equiv \frac{\left(\frac{\left(g_{\sigma B_{8} B_{8}}^{2}\right)_{Q^{2}=0}}{\left(g_{\sigma N N}^{2}\right)_{Q^{2}=0}}\right)}{\left(\frac{\left(g_{\sigma B_{8} B_{8}}^{2}\right)_{Q^{2}=M_{\sigma}^{2}}}{\left(g_{\sigma N N}^{2}\right)_{Q^{2}=M_{\sigma}^{2}}}\right)} .
$$

By using the average value $\Lambda_{\sigma N N}=3.97 \mathrm{fm}^{-1}$, Eq. (47), we get

$$
\begin{aligned}
\Lambda_{\sigma N N} & =3.97 \mathrm{fm}^{-1} \\
\Lambda_{\sigma \Lambda \Lambda} & =4.48 \mathrm{fm}^{-1} \\
\Lambda_{\sigma \Sigma \Sigma} & =4.75 \mathrm{fm}^{-1} \\
\Lambda_{\sigma \Xi \Xi} & =3.45 \mathrm{fm}^{-1} .
\end{aligned}
$$

Thus we can use $\Lambda_{\sigma B_{8} B_{8}} \simeq 4.0 \mathrm{fm}^{-1}$ as an average value for the whole baryon octet. 


\section{C. $a_{0} \mathbf{B}_{8} \mathbf{B}_{8}$}

The results obtained for $\sigma$ can be extrapolated to the $a_{0}$ meson in a straightforward way. Let us recall that in the additive constituent quark pattern one has degenerate masses $M_{a_{0}}=M_{\sigma}$ (see Ref. [11] for an explanation of the non-degeneracy). Then by writing the $a_{0}$ on-shell couplings in SU(3) language

$$
\begin{aligned}
\left(g_{a_{0} N N}\right)_{Q^{2}=M_{a_{0}}^{2}} & =g_{s,+} \\
\left(g_{a_{0} \Sigma \Sigma}\right)_{Q^{2}=M_{a_{0}}^{2}} & =2 g_{s,+} \alpha_{s} \\
\left(g_{a_{0} \Xi \Xi}\right)_{Q^{2}=M_{a_{0}}^{2}} & =g_{s,+}\left(2 \alpha_{s}-1\right),
\end{aligned}
$$

and imposing the degeneracy we predict from our value for $g_{s,+}$ (Eq. (80))

$$
\begin{aligned}
& \frac{\left(g_{a_{0} N N}^{2}\right)_{Q^{2}=M_{a_{0}}^{2}}}{4 \pi}=\frac{\left(g_{a_{0} \Xi \Xi}^{2}\right)_{Q^{2}=M_{a_{0}}^{2}}}{4 \pi}=7.6 \\
& \frac{\left(g_{a_{0} \Sigma \Sigma}^{2}\right)_{Q^{2}=M_{a_{0}}^{2}}}{4 \pi}=30.3 .
\end{aligned}
$$

\section{D. $\pi \mathbf{B}_{8} \mathbf{B}_{8}$}

In order to avoid mass factors we shall use the pseudovector coupling for the pion, i.e., the lagrangian

$$
\tilde{\mathcal{L}}_{\pi q q}^{P V}=-\frac{\tilde{f}_{\pi q q}}{M_{\pi}} \bar{q} i \gamma_{5} \gamma_{\mu} \vec{\tau} \partial^{\mu} \vec{\pi} q
$$

with the vertex form factor

$$
\tilde{F}_{\pi q q}\left(Q^{2}\right) \equiv\left(\frac{\tilde{\Lambda}^{2}-M_{\pi}^{2}}{\tilde{\Lambda}^{2}-Q^{2}}\right)^{1 / 2}
$$

to derive the asymptotic pion exchange central potentials. To perform the calculation we choose a total (spin, isospin) in each case. By considering the ${ }^{1} S_{0}$ partial wave for instance we take $(0,1)$ for $N N$ and $\Xi \Xi$ and $(0,0)$ for $\Sigma \Sigma$ interactions. Then we have

$$
\begin{aligned}
\left(V_{q}\right)_{\mathrm{OPE}}^{N N \rightarrow N N}(R \rightarrow \infty) & =\frac{\tilde{f}_{\pi q q}^{2}}{4 \pi}\left[-\frac{75}{9} e^{\frac{M_{\pi}^{2} \tilde{b}^{2}}{2}}\right] \frac{1}{3} \frac{e^{-M_{\pi} R}}{R} \\
\left(V_{q}\right)_{\mathrm{OPE}}^{\Sigma \Sigma \rightarrow \Sigma \Sigma}(R \rightarrow \infty) & =\frac{\tilde{f}_{\pi q q}^{2}}{4 \pi}\left[\frac{32}{3} e^{\frac{M_{\pi}^{2} \tilde{b}^{2}}{2}}\right] \frac{1}{3} \frac{e^{-M_{\pi} R}}{R} \\
\left(V_{q}\right)_{\mathrm{OPE}}^{\Xi \Xi \Xi \Xi}(R \rightarrow \infty) & =\frac{\tilde{f}_{\pi q q}^{2}}{4 \pi}\left[-\frac{3}{9} e^{\frac{M_{\pi}^{2} \tilde{b}^{2}}{2}}\right] \frac{1}{3} \frac{e^{-M_{\pi} R}}{R} .
\end{aligned}
$$

On the other hand at the baryonic level the lagrangians can be expressed as

$$
\begin{aligned}
& \mathcal{L}_{\pi N N}^{P V}=-\frac{\left(f_{\pi N N}\right)_{Q^{2}=M_{\pi}^{2}}}{M_{\pi}} i \gamma_{5} \gamma_{\mu} \vec{\tau} \partial^{\mu} \vec{\pi} N \\
& \mathcal{L}_{\pi \Sigma \Sigma}^{P V}=-\frac{\left(f_{\pi \Sigma \Sigma}\right)_{Q^{2}=M_{\pi}^{2}}}{M_{\pi}}(\vec{\Sigma} \times \vec{\Sigma}) \gamma_{5} \gamma_{\mu} \partial^{\mu} \vec{\pi}
\end{aligned}
$$




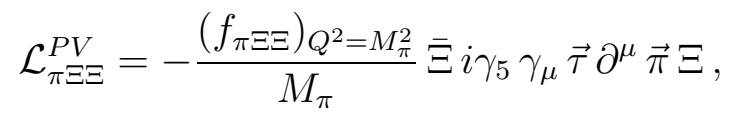

with form factors

$$
\begin{aligned}
G_{\pi N N}\left(Q^{2}\right) & =\left(\frac{\Lambda_{\pi N N}^{2}-M_{\pi}^{2}}{\Lambda_{\pi N N}^{2}-Q^{2}}\right)^{\frac{1}{2}} \\
G_{\pi \Sigma \Sigma}\left(Q^{2}\right) & =\left(\frac{\Lambda_{\pi \Sigma \Sigma}^{2}-M_{\pi}^{2}}{\Lambda_{\pi \Sigma \Sigma}^{2}-Q^{2}}\right)^{\frac{1}{2}} \\
G_{\pi \Xi \Xi}\left(Q^{2}\right) & =\left(\frac{\Lambda_{\pi \Xi \Xi}^{2}-M_{\pi}^{2}}{\Lambda_{\pi \Xi \Xi}^{2}-Q^{2}}\right)^{\frac{1}{2}},
\end{aligned}
$$

and where in conventional SU(3) notation

$$
\begin{aligned}
\left(f_{\pi N N}\right)_{Q^{2}=M_{\pi}^{2}} & =f_{p,+} \\
\left(f_{\pi \Sigma \Sigma}\right)_{Q^{2}=M_{\pi}^{2}} & =2 f_{p,+} \alpha_{p} \\
\left(f_{\pi \Xi \Xi}\right)_{Q^{2}=M_{\pi}^{2}} & =-f_{p,+}\left(1-2 \alpha_{p}\right),
\end{aligned}
$$

having introduced $f_{p,+} \equiv\left(f_{p, D}+f_{p, F}\right)$ as the sum of the symmetric and antisymmetric pseudoscalar octet coupling constants, and $\alpha_{p} \equiv f_{P, F} /\left(f_{P, D}+f_{P, F}\right)$ as the $F /(F+D)$ ratio of the pseudoscalar octet.

By using the same total (spin, isospin) channels as above the corresponding asymptotic pion exchange central potentials are

$$
\begin{aligned}
\left(\mathcal{V}_{B}\right)_{\mathrm{OPE}}^{N N \rightarrow N N}(R \rightarrow \infty) & =\frac{\left(f_{\pi N N}^{2}\right)_{Q^{2}=M_{\pi}^{2}}}{4 \pi}[-3] \frac{1}{3} \frac{e^{-M_{\pi} R}}{R} \\
\left(\mathcal{V}_{B}\right)_{\mathrm{OPE}}^{\Sigma \Sigma \rightarrow \Sigma \Sigma}(R \rightarrow \infty) & =\frac{\left(f_{\pi \Sigma \Sigma}^{2}\right)_{Q^{2}=M_{\pi}^{2}}}{4 \pi}[6] \frac{1}{3} \frac{e^{-M_{\pi} R}}{R} \\
\left(\mathcal{V}_{B}\right)_{\mathrm{OPE}}^{\Xi \Xi \rightarrow \Xi \Xi}(R \rightarrow \infty) & =\frac{\left(f_{\pi \Xi \Xi}^{2}\right)_{Q^{2}=M_{\pi}^{2}}}{4 \pi}[-3] \frac{1}{3} \frac{e^{-M_{\pi} R}}{R} .
\end{aligned}
$$

Then from the comparison of the asymptotic potentials at the baryon level and quark level the following relations come out

$$
\begin{aligned}
\left(f_{\pi N N}^{2}\right)_{Q^{2}=M_{\pi}^{2}} & =\tilde{f}_{\pi q q}^{2} \frac{25}{9} e^{\frac{M_{\pi}^{2} \tilde{b}^{2}}{2}} \\
\left(f_{\pi \Sigma \Sigma}^{2}\right)_{Q^{2}=M_{\pi}^{2}} & =\tilde{f}_{\pi q q}^{2} \frac{16}{9} e^{\frac{M_{\pi}^{2} \tilde{b}^{2}}{2}} \\
\left(f_{\pi \Xi \Xi}^{2}\right)_{Q^{2}}=M_{\pi}^{2} & =\tilde{f}_{\pi q q}^{2} \frac{1}{9} e^{\frac{M_{\pi}^{2} \tilde{b}^{2}}{2}}
\end{aligned}
$$

and

$$
\begin{aligned}
& \frac{\left(f_{\pi \Sigma \Sigma}^{2}\right)_{Q^{2}=M_{\pi}^{2}}}{\left(f_{\pi N N}^{2}\right)_{Q^{2}=M_{\pi}^{2}}}=\frac{16}{25} \\
& \frac{\left(f_{\pi \Xi \Xi \Xi}^{2}\right)_{Q^{2}=M_{\pi}^{2}}}{\left(f_{\pi N N}^{2}\right)_{Q^{2}=M_{\pi}^{2}}}=\frac{1}{25} .
\end{aligned}
$$




\section{Coupling constants and form factors values}

From Eqs. (99) and the standard value $\left(f_{\pi N N}^{2}\right)_{Q^{2}=M_{\pi}^{2}} / 4 \pi=0.079$ we predict the numerical values

$$
\begin{aligned}
& \frac{\left(f_{\pi \Sigma \Sigma}^{2}\right)_{Q^{2}=M_{\pi}^{2}}}{4 \pi}=0.051 \\
& \frac{\left(f_{\pi \Xi \Xi}^{2}\right)_{Q^{2}=M_{\pi}^{2}}}{4 \pi}=0.0032
\end{aligned}
$$

or

$$
\begin{aligned}
& \frac{\left(g_{\pi \Sigma \Sigma}^{2}\right)_{Q^{2}=M_{\pi}^{2}}}{4 \pi}=9.4 \\
& \frac{\left(g_{\pi \Xi \Xi}^{2}\right)_{Q^{2}=M_{\pi}^{2}}}{4 \pi}=0.6
\end{aligned}
$$

and

$$
\alpha_{p}=0.4 \text {. }
$$

This compares quite well with a derived value of $\alpha_{p} \simeq 0.365 \pm 0.007$ from the $F / D$ ratio extracted from semileptonic decays of baryons [20].

With respect to the couplings at $Q^{2}=0$ we can rely on the quite approximate Goldstone boson character of the pion and assume they are given by $\left(f_{\pi B_{8} B_{8}}^{2}\right)_{Q^{2}=0} \simeq\left(f_{\pi B_{8} B_{8}}^{2}\right)_{Q^{2}=M_{\pi}^{2} \rightarrow 0}$. As in this limit the ratios between the couplings are the same than the on-shell ones obtained above, Eq. (99), we can use them altogether with $\left(f_{\pi N N}^{2}\right)_{Q^{2}=M_{\pi}^{2} \rightarrow 0} / 4 \pi=0.072$, Eq. (35), to predict

$$
\begin{aligned}
& \frac{\left(f_{\pi \Sigma \Sigma}^{2}\right)_{Q^{2}=0}}{4 \pi} \simeq 0.046 \\
& \frac{\left(f_{\pi \Xi \Xi}^{2}\right)_{Q^{2}=0}}{4 \pi} \simeq 0.0029
\end{aligned}
$$

or

$$
\begin{aligned}
& \frac{\left(g_{\pi \Sigma \Sigma}^{2}\right)_{Q^{2}=0}}{4 \pi} \simeq 8.5 \\
& \frac{\left(g_{\pi \Xi \Xi}^{2}\right)_{Q^{2}=0}}{4 \pi} \simeq 0.5 .
\end{aligned}
$$

Moreover the preservation of the ratios implies the equality of the form factors. From $\left(f_{\pi N N}^{2}\right)_{Q^{2}=0} \simeq\left(f_{\pi N N}^{2}\right)_{Q^{2}=M_{\pi}^{2} \rightarrow 0}$ and $\left(f_{\pi N N}^{2}\right)_{Q^{2}=M_{\pi}^{2}}$ we deduce

$$
\Lambda_{\pi B_{8} B_{8}} \simeq 2.35 \mathrm{fm}^{-1}
$$

\section{SUMMARY}

From a $\mathrm{SU}(2) \otimes \mathrm{SU}(2)$ chiral quark lagrangian incorporating spontaneous chiral symmetry breaking, and its generalization to $\mathrm{SU}(3) \otimes \mathrm{SU}(3)$, asymptotic meson exchange $B_{8} B_{8} \rightarrow B_{8} B_{8}$ interaction potentials are derived in the Born-Oppenheimer approximation. The comparison 
with the corresponding potentials from a $\mathrm{SU}(2)$ or $\mathrm{SU}(3)$ invariant hadronic lagrangian allows for the expression of the $\pi B_{8} B_{8}$ and $\sigma B_{8} B_{8}$ coupling constants in terms of the elementary $\pi q q$ and $\sigma q q$ ones. By using the $\pi N N$ coupling constant as an input the $\pi q q$ one gets fixed. From it the rest of coupling constants $\left(\sigma q q, \pi B_{8} B_{8}\right.$ and $\left.\sigma B_{8} B_{8}\right)$ are predicted. Their $M_{m} \rightarrow 0$ limits are also of interest to be compared with the values of the couplings at $Q^{2}=0$ provided by phenomenological analyses or QCDSR. The similar value obtained for $\pi N N$ indicates the quite approximate Goldstone boson nature of the pion. On the contrary $\sigma B_{8} B_{8}$ couplings are significantly different as might be expected.

Further information about the couplings can be extracted under the assumption that our on-shell model predictions and the $Q^{2}=0$ values from external analyses can be managed jointly. Though this assumption is debatable it allows to get some insight into the cutoff parameters $\Lambda_{m B_{8} B_{8}}$ at the baryonic level.

TABLE II: Pion and sigma coupling constants to quarks in our $\mathrm{SU}(2) \otimes \mathrm{SU}(2)$ and $\mathrm{SU}(3) \otimes \mathrm{SU}(3)$ models.

\begin{tabular}{ccc}
\hline \hline$m$ & $\mathrm{SU}(2) \otimes \mathrm{SU}(2)$ & $\mathrm{SU}(3) \otimes \mathrm{SU}(3)$ \\
\hline$(\pi, \sigma)$ & $g_{c h}^{2} / 4 \pi=0.535$ & $\tilde{g}_{c h}^{2} / 4 \pi=0.535$ \\
$\pi$ & $g_{\pi q q}^{2} / 4 \pi=0.55$ & $\tilde{g}_{\pi q q}^{2} / 4 \pi=0.54$ \\
$\sigma$ & $g_{\sigma q q}^{2} / 4 \pi=1.59$ & $\tilde{g}_{\sigma q q}^{2} / 4 \pi=0.93$ \\
\hline \hline
\end{tabular}

We summarize in Table the pion and sigma coupling constants to quarks in our models. In Table III the values obtained for the coupling constants and the form factors parameters at the baryon level are listed. By making use of the $a_{0}-\sigma$ degeneracy in our quark

TABLE III: Predicted pairs $\left(\left(g_{m B_{8} B_{8}}^{2} / 4 \pi\right)_{Q^{2}=M_{m}^{2}}, \Lambda_{m B_{8} B_{8}}\left(f m^{-1}\right)\right)$ from the chiral quark potential model, for exact $\mathrm{SU}(3)$ symmetry $\left(M_{\Lambda}=M_{\Sigma}=M_{\Xi}=M_{N}=939 \mathrm{MeV}\right)$. The superindex * indicates the $\pi N N$ coupling constant value used as input.

\begin{tabular}{|c|c|c|c|c|}
\hline$m$ & $m N N$ & $m \Lambda \Lambda$ & $m \Sigma \Sigma$ & $m \Xi \Xi$ \\
\hline & $\left.4.6^{*}, 2.35\right)$ & & $(9.4,2.35)$ & $(0.6,2.35)$ \\
\hline$\sigma$ & $8.2,3.97)$ & $(30.3,4.48)$ & $(30.3,4.75)$ & $(7.6,3.45)$ \\
\hline
\end{tabular}

model we have also predicted $a_{0} B_{8} B_{8}$ on-shell couplings. Concerning other diagonal $m B B$ couplings such as $f_{0} B_{8} B_{8}, \eta B_{8} B_{8}$ and $\eta^{\prime} B_{8} B_{8}$ to which our formalism could be also applied the situation gets complicated by the presence of the strange quark and/or antiquark which may give rise to relevant $\mathrm{SU}(3)$ breaking effects out of the scope of our symmetry treatment.

Let us finally add that in our model Goldberger-Treiman relations of the form $\left(g_{A}\right)_{\pi B_{8} B_{8}} / 2 f_{\pi}=\left(f_{\pi B_{8} B_{8}}\right)_{Q^{2}=M_{\pi}^{2}} / M_{\pi}$, where $g_{A}$ stands for the axial coupling constant and $f_{\pi}$ for the pion decay constant, can be immediately applied. In our non-relativistic description $\left(g_{A}\right)_{\pi N N}=5 / 3$, a value considerable larger than the experimental one 1.267. Consequently $f_{\pi}=116 \mathrm{MeV}$, which is $20 \%$ bigger than the experimental value of $93 \mathrm{MeV}$. Regarding these discrepancies it has been shown [18] that a relativistic treatment, beyond our present approach, could correct them to a good extent. 


\section{Acknowledgments}

This work has been partially funded by Ministerio de Educación y Ciencia and EU FEDER under Contract No. FPA2007-65748, by Junta de Castilla y León under Contract No. SA016A17, and by the Spanish Consolider-Ingenio 2010 Program CPAN (CSD200700042).

[1] T.E.O. Ericson, Nucl. Phys. A 543, 409c (1992). D.V. Bugg, Eur. Phys. J. C 33, 505 (2004).

[2] V.G.J. Stocks and Th.A. Rijken, Nucl. Phys. A 613, 311 (1997).

[3] A. Valcarce, H. Garcilazo, F. Fernández, and P. González, Rep. Prog. Phys. 68, 965 (2005) and references therein.

[4] Z.Y. Zhang et al., Nucl. Phys. A 625, 59 (1997).

[5] K. Shimizu, S. Takeuchi, and A. Buchmann, Prog. Theor. Phys. Supp. 137, 43 (2000).

[6] Y. Fujiwara, Y. Suzuki, and C. Nakamoto, Prog. Part. Nucl. Phys. 58, 439 (2007).

[7] G.Q. Liu, M. Swift, and A.W. Thomas, Nucl. Phys. A 556, 331 (1993).

[8] D.I. Diakonov and V.Yu. Petrov, Nucl. Phys. B 272, 457 (1986).

[9] F. Fernández and E. Oset, Nucl. Phys. A 455, 720 (1986).

[10] A.M. Kusainov, V.G. Neudatchin, and I.T. Obukhovsky, Phys. Rev. C 44, 2343 (1991).

[11] M.D. Scadron, Phys. Rev. D 26, 239 (1982).

[12] W.Y. Hwang, Z. Phys. C 75, 701 (1997).

[13] E.M. Henley and G.A. Miller, Phys. Lett. B 251, 453 (1991).

[14] A.W. Thomas and K. Holinde, Phys. Rev. Lett. 63, 2025 (1989).

[15] G. Erkol, R.G.E. Timmermans, and Th.A. Rijken, Phys. Rev. C 72, 035209 (2005).

[16] J. Carlson, J. Kogut, and V.R. Pandharipande, Phys. Rev. D 27, 233 (1983). J.L. Basdevant and S. Boukraa, Z. Phys. C 30, 103 (1986). F. Cano, P. González, S. Noguera, and B. Desplanques, Nucl. Phys. A 603, 257 (1996). H. Garcilazo and A. Valcarce, Phys. Rev. C 68, 035207 (2003).

[17] B. Juliá-Díaz, A. Valcarce, P. González, and F. Fernández, Phys. Rev. C 66, 024005 (2002).

[18] B. Juliá-Díaz, D. O. Riska and F. Coester, Phys. Rev. C 70, 045204 (2004).

[19] G. Erkol, R.G.E. Timmermans, M. Oka, and Th.A. Rijken, Phys. Rev. C 73, 044009 (2006).

[20] F.E. Close and R.G. Roberts, Phys. Lett. B 316, 165 (1993). 